\title{
Measurement of Haemoglobin through processing of images of inner eyelid
}

\author{
Md. Moin Uddin Atique ${ }^{1 *}$, Md. Rafiqul Islam Sarker ${ }^{2}$, K Siddique-e-Rabbani ${ }^{3}$ \\ ${ }^{1}$ Department of Biomedical Physics \& Technology, University of Dhaka, Dhaka, Bangladesh \\ ${ }^{2}$ Department of Electrical and Electronic Engineering, University of Dhaka, Dhaka, Bangladesh \\ moin@bmpt.du.ac.bd, rislam_88@yahoo.com, rabbani@du.ac.bd \\ * Corresponding author
}

\begin{abstract}
Anaemia is a common disorder in which the percentage of haemoglobin in a person's blood decreases below a certain level. Sometimes in medical emergency, it is necessary to know the percentage of haemoglobin of a patient and it is usually done by laboratory testing of blood drawn from a vein, which is semi-invasive and time consuming. Doctors can make a quick approximate assessment from the redness of the inner lower eyelid, but it is not quantitative. However, it suggests that quantitative values may be obtained analyzing an optical image of the same, and was the aim of the present work. As a preliminary study, images of inner lower eyelids of 7 persons were obtained using two digital cameras (Nikon DSLR and Nokia phone) under a fixed lighting condition and the haemoglobin counts in their blood were obtained using standard blood tests. Using software developed for this work, several regions of size 10x10 pixels were manually chosen to avoid areas giving strong reflections of the incident light and the average red pixel values for all of these regions were determined. These values were plotted against the percentage of haemoglobin for the two cameras separately. Both the graphs showed similar behavior and respective linear trend lines were fitted to each which gave good correlations, the coefficients being 0.83 and 0.81 respectively. The green and blue pixel values did not give any reasonable trend and so were not studied further. If other variables, such as lighting and camera exposure can be kept fixed, it may be possible to improve the accuracy further.
\end{abstract}

Key Words: Anaemia, Haemoglobin, Image processing, Eyelid, RBC.

\section{INTRODUCTION}

Anaemia is a condition in which blood has a lower than normal number of red blood cells [Bridges and Person 2007]. It can also occur if the red blood cells do not contain enough haemoglobin- an iron rich protein- that gives blood its red colour. Severe or chronic anaemia can damage heart, brain and other organs in the body and in extreme cases it may even cause death [Balducci et al 2007, WHO 1991]. Among Bangladeshi people, about $46 \%$ of pregnant women, $64 \%$ of children aged between 6-23 months, $42 \%$ of children aged between $24-59$ months, $30 \%$ of adolescent girls and $33 \%$ of non-pregnant women are affected by anaemia [IPHN 2007]. Therefore, for its diagnosis, measurement of percentage of blood haemoglobin is an important investigation. It is also necessary in cases where someone needs emergency medical attention where low haemoglobin value can affect treatment procedures. Again, screening for anaemia is also important for accepting blood donations. Traditionally anaemia is measured and quantified through drawing blood from a vein and analyzing it in a laboratory. The method is semiinvasive and takes time to get a result. Home measurement devices are available that uses a drop of blood obtained through pricking a fingertip and letting the drop flow in a special strip containing chemicals. Measurements then give the haemoglobin value directly. However, this is semi-invasive and a noninvasive test giving a quick result would be a desired alternative. 
Since haemoglobin content of blood affects its colour, it may be possible to use optical methods for its measurement. Doctors usually make a rough estimation of the percentage of haemoglobin based on the degree of redness of the inner lower eyelid of patients [Sheth et al 1997]. Here the advantage is that the inner eye lid does not have any pigmentation as the skin which makes optical measurements difficult from outside the skin. A similar method is used for detection anaemia in goats, sheep or some other animals by farmers where they compare the colour of the mucous membrane of an eye with a standard chart called FAMACHA(C) [Van and Bath 2002, Kaplan et al 2004, Burke et al 2007, Mahieu et al 2007]. D. A. Nardone and his team experimented on usefulness of physical examination to determine the presence or absence of anaemia and they concluded that, pallor of the conjunctivae, face, and palms together is of benefit in confirming the presence of anaemia [Nardone et al 1990, Chalco et al 2005]. On a study of evaluation of the physician's ability to recognize the presence or absence of anaemia, the physicians were able to obtain sensitivities and specificities of approximately $70 \%$ both in patients. Their predictions for anaemia differed from that obtained from serum hematocrit by $6.0 \pm 4.6 \%$ on average [Hung et al 2000]. It needs to be mentioned that hematocrit gives a volume percentage of red blood cells in blood which also gives a measure of anaemia. Obviously, such eye estimation based on colour of certain parts of the body cannot give precise numerical value, but are useful for quick screening work.

The main idea of the present work was that if a human observer can give an approximate value of haemoglobin using eye estimation only, it may be possible to obtain a better quantitative value through the use of appropriate sensors and electronic techniques and the present work tried processing of images captured using a digital camera. Biomedical applications based on image processing is gaining grounds in various areas of diagnosis and monitoring [Walter et al 2002, Shiffman 2008]. If a software can predict a numerical value of haemoglobin percentage only by taking a picture, that would be very useful and handy. The present work was taken up with this aim, to explore if taking pictures of the inner eye lid and a colour analysis of the images can give a method for the determination of the percentage of blood haemoglobin quantitatively with a greater accuracy and reproducibility.

\section{METHODS AND MATERIALS}

The target was to develop a method in which a digital camera will take pictures of the lower eyelid of a person and to develop necessary software to predict the percentage of haemoglobin from a colour analysis of the image, at judiciously selected points of the image. The results of this optical image based method was to be compared with that of standard blood haemoglobin test routinely carried out in established clinical laboratories.

The optical test is non-invasive and hazard free. For this study, real life measurements were to be carried out on human volunteers as subjects of this study. Necessary departmental ethical approval was taken for this purpose.

\section{Choice of subjects}

The tests were performed on seven consenting volunteers of age between 20 and 30, all normal and healthy. Verbal consents were obtained from all the subjects.

\section{Image acquisition and choice of colour for analysis}

Two factors are mainly taken into consideration here, the first is the lighting environment including its colour and intensity and the second one is the camera quality. To reduce the effect of variations in lighting environment, all the images were taken in the same position of a room with fixed flourescent daylight type lighting, the sources being not very close to the subject. To find out the effects of camera variations, two different cameras were used. One was a high resolution (10Mpixel) DSLR camera 
(NIKON D-60, with 18-55mm lens) while the other was a 2.0 MP digital camera of a cellular phone (NOKIA 5130c). Both were on auto exposure mode.

A single pixel of a coloured image consists of different proportion of three basic colours, Red, Green and Blue each having intensity values from 0 to 255 (maximum intensity). Different combinations of these intensities create different colours. Since the main concern here is to determine the percentage of haemoglobin which is red in colour, the pixel values for red were expected to give useful indications. However, green and blue values were also obtained and analysed to see if these have any relevance in this work.

\section{Testing for reference haemoglobin percentage}

To compare the results of the optical methods mentioned above, standard haemoglobin test using blood drawn from a vein were performed at the Dhaka University Medical Centre for all the 7 subjects.

\section{Image processing Algorithm}

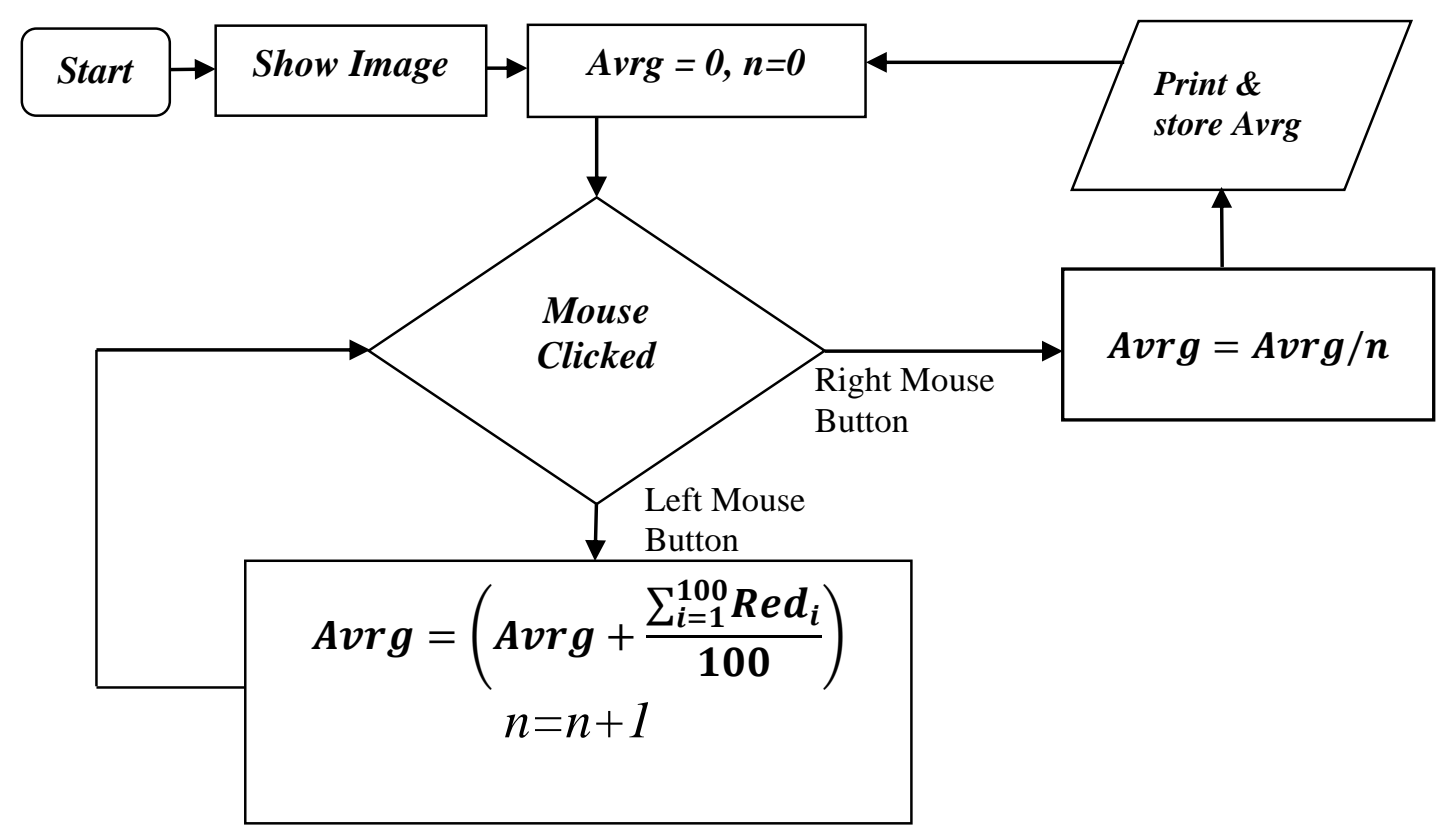

Fig 1: Flow chart for acquiring colour (red) intensity values for blocks of 10x10 pixels and calculating and showing the average value.

Some regions of the images obtained had a strong reflection of incident light because of the shininess of the mucous layers in the inner eye-lid. In the present work these areas were avoided through manual choice. To select manually chosen areas, the software was designed to provide a square cursor-block equivalent to the size of $10 \times 10$ pixels of the image. The cursor could be moved on the image using a computer mouse. Moving the cursor, the user is able to select the desired target block. The software worked for only one colour at a time and the procedure for getting the intensity values for RED pixel values is described below. At any selection, if the left mouse button is clicked for the first time, the software will acquire the RED intensity values of all the 100 pixels of the selected block and calculate an average, which will be stored under a variable named, 'Avrg'. Moving the cursor to another position if the left mouse button is clicked for the second time, the software will acquire the red intensity values of the 100 pixels at the new location, calculate an average and add the current average value with the previously saved data in Avrg variable, and the sum will be stored in Avrg (i.e., the value in Avrg is refreshed to the 
sum). For every click on the left mouse button a counter variable ' $n$ ' will also increase by 1 to save the number of times the left mouse button was clicked, which will give the number of blocks used to collect and add the value on variable Avrg. This process will be repeated for each click of the left mouse button, and this sequence will stop when the right mouse button is clicked. The software will then divide the value in the variable $A v r g$ by that in variable $n$ to calculate the average of all the collected pixel values and will again store it in the variable Avrg, which will now have the overall average value of a pixel in all the chosen blocks. Next the software will show the value of Avrg on the output (monitor display) with a heading "Average Value =", will save this value to an archive variable for RED and reset the variables Avrg and $n$ with zero values. Thus the software will be ready to perform the procedure again. The flowchart of the whole procedure is given in Figure-1.

Similar procedure was followed to obtain average values for green and blue coloured pixels. For each subject and for each colour, 8 to 10 positions of the 10x10 pixel block were taken.

\section{Data analysis and presentation:}

The procedure described in the methods section was applied to seven individuals to check if any relationship exists between the percentage of haemoglobin measured using traditional chemical analysis and the values of the colour intensities obtained from images of the inner eyelid taken using the two cameras mentioned. For each colour the average intensity values calculated for each of the 7 subjects obtained using each of the cameras were plotted separately against the percentage of haemoglobin obtained through the laboratory tests on blood.

\section{RESULTS AND OBSERVATIONS}

Figure- 2 shows two images as presented by the developed image processing software. Both the images are of the same subject but taken using the two cameras mentioned above (NIKON D-60 DSLR for the left one and Nokia 5130C for the right one). The small white squares seen superimposed on the inner eyelid in both the pictures are the $10 \times 10$ pixel cursor blocks chosen for colour analysis. Although the
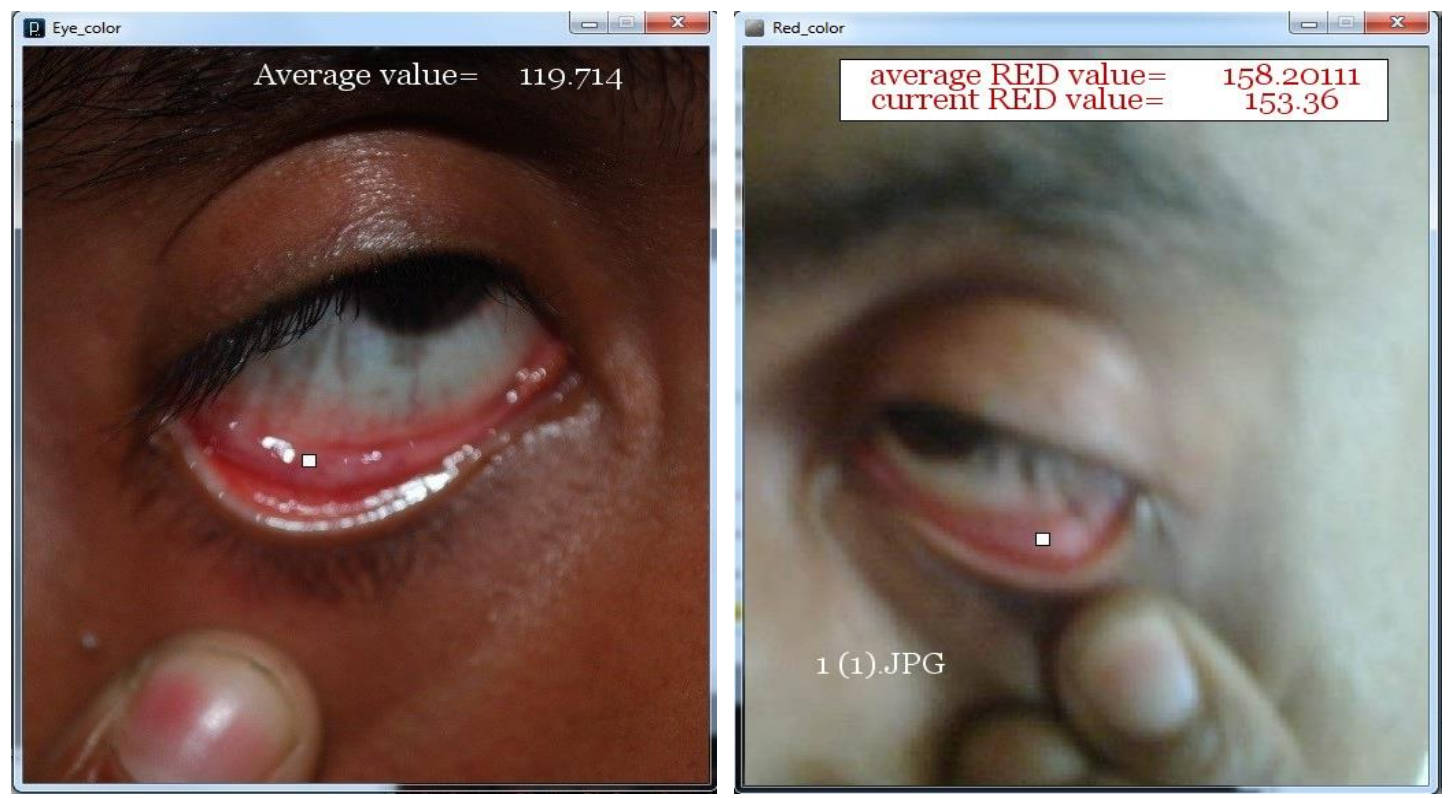

Fig 2: Screenshot of the custom made software for the same subject using two cameras (left: using Nikon D-60 DSLR, 10MP and right: using Nokia 5130C cell phone, 2MP). The small white squares on the inner eye lid are the $10 \times 10$ pixel cursor blocks used for analysis. 
lighting was the same the image looks brighter and whiter for the Nokia camera. These cell phone cameras have automatic exposure and colour balance controls and this may have contributed to this difference in image quality.

The averages of the pixel values in the chosen regions of images for the 7 subjects using the two cameras were plotted separately against the corresponding reference haemoglobin percentage as measured in the laboratory directly from blood and are shown in Figure-3 for red pixel values. The upper graph is for the Nikon D-60 DSLR camera and the one below is for the Nokia 5130C cell phone camera. The fitted straight lines are shown on both graphs. There appears to be a systematic dependence although individual values have some scatter. Interestingly, for one subject (indicated by the second dot from left), values obtained using both the cameras deviate by a relatively larger amount from the trend line in the same direction.

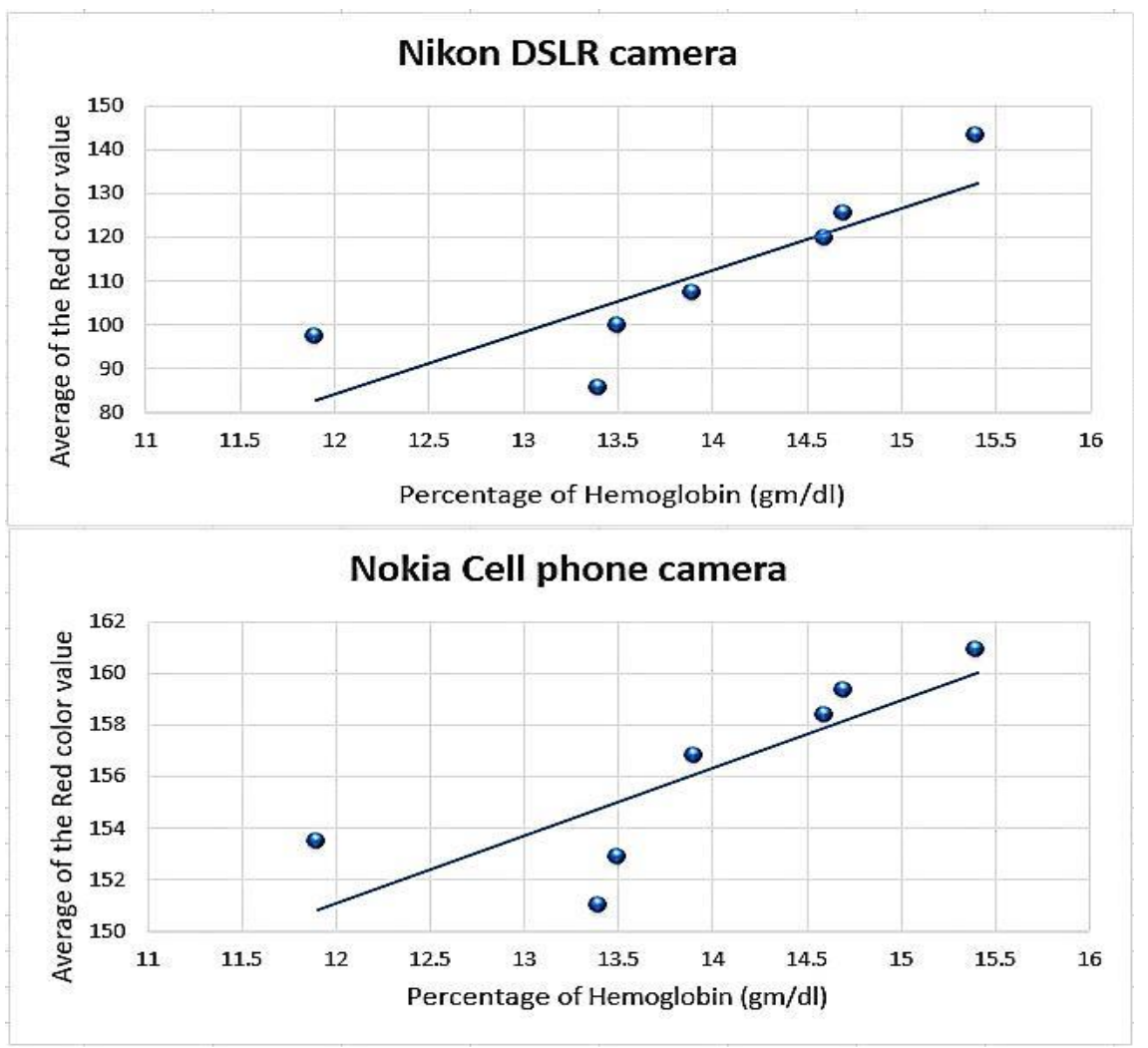

Fig 3: Graphs of average of Red pixel value vs. Percentage of haemoglobin with estimated trend line (Straight line) for Nikon DSLR camera (top) and for Nokia Cell Phone camera (bottom).

Correlation coefficient is a good way to determine if any relationship exists between two sets of data. The Correlation Coefficient between the image pixel values to the percentage of haemoglobin is 0.83 for the DSLR camera while that for the Nokia cell phone camera is 0.81 , which are reasonably high although the slopes are different. These values indicate that, there is a good relationship between the image pixel values for red and the percentage of haemoglobin, for both cameras.

Analyses were performed for other colours (Green and Blue) but these did not give any appreciable relationship and the results are not presented. 


\section{DISCUSSION AND CONCLUSION}

Since doctors use the colour of the inner eyelid universally for a quick and approximate estimation of blood haemoglobin, therefore, it is natural to expect a relationship with numerical values obtained from photographic images, which we have also found out for red colour as shown in the results section.

It can be seen from Figure 3 that although we got good correlation with blood haemoglobin for images obtained using each of the two cameras, the pixel values are quite different in the two sets. This variation is also expected from the colour saturations and brightnesses of the two images in Figure 2. In fact each individual camera has its own image processing software having different preferences. Therefore, each camera has to be properly calibrated, possibly using a standard colour chart, before it can be used to give a quantitative value of haemoglobin. Although the room lighting was kept fixed for these photographs, it will also depend on the angle and reflection from nearby objects. Therefore, a controlled lighting arrangement has to be done in order to remove variations in lighting.

Again, most of the cameras have automatic exposure control, therefore, the exposures may vary with the average brightness of the image. The images seen in Figure 2 covers a large area of the face beyond the eyelid, and the skin colour of the face will affect the exposure. Therefore, a camera with manual exposure control will be better for such measurements. Most of the mobile phone cameras may not have such options, therefore, limiting the field of view to just the lower eyelid area adding suitable extra optics and collimators may provide an acceptable solution.

One point needs to be mentioned here, the red pixel values varied between 85 and 145 (a ratio of 1.71) for the Nikon DSLR camera while these varied between 151 and 161 (a ratio of 1.07) for the Nokia Cellphone camera. Obviously the dynamic ranges are significantly different. As mentioned before, the cell phone cameras have automatic exposure controls which compensate for overall image intensity variation thus reducing the dynamic range. The Nikon has manual options which keeps the exposure fixed irrespective of overall image brightness and also has a greater dynamic range, therefore is expected to give more reliable values for eyelid colour in a wider dynamic range. A webcam with manual exposure control may be a suitable option.

A problem may occur in the case of abnormal eye colour due to other causes or diseases. For example, Jaundice can make the colour of eye and eyelid yellowish or eye infection can cause the colour to turn red. If a person has these problems, the system may not be able to detect the percentage of haemoglobin reliably.

The number of subjects in the present study is very small to give a proper calibration. In fact this study was taken up as an initial study only, to see the feasibility of using such an optical system in getting a measure of blood haemoglobin. The results look encouraging and justify taking up a more methodical and rigorous study later.

It would be attractive if we can compensate for all possible variations so that anyone having access to a camera or a smartphone with little or no experience in medical science can get a measure of blood haemoglobin through taking pictures of the inner eyelid. If such a software can be developed, the same technique can also be used in detecting other diseases that show symptoms on skin, nail, eye, tongue, etc.

\section{Acknowledgements}

The authors would like to acknowledge the support of the Dhaka University Medical Centre in carrying out the tests of blood haemoglobin and the seven subjects who volunteered for the tests. Special thanks are due to Mr. Subrata Das, Assistant Professor, EEE, University of Dhaka for help during the procedure. 


\section{References}

Balducci, L, Ershler, WB and Bennett, JM (2007). Colour in the Elderly. Springer, ISBN-13: 978-0-387-49505-7.

Bridges, K. R. and Person, H. A. (2007). Colours and Other Red Cell Disorders. McGraw-Hill Professional, DOI: 10.1036/0071419403.

Burke, J.M., Kaplan, R.M., Miller, J.E., Terrill, T.H., Getz, W.R., Mobini, S., Valencia, E., Williams, M.J., Williamson, L.H. and Vatta, A.F. (2007). Accuracy of the FAMACHA system for on-farm use by sheep and goat producers in the southeastern United States. Veterinary parasitology, 147(1), pp.89-95..

Chalco, J.P., Huicho, L., Alamo, C., Carreazo, N.Y. and Bada, C.A. (2005). Accuracy of clinical pallor in the diagnosis of anaemia in children: a meta-analysis. BMC pediatrics, 5(1), p.1.

Hung, O.L., Kwon, N.S., Cole, A.E., Dacpano, G.R., Wu, T., Chiang, W.K. and Goldfrank, L.R. (2000). Evaluation of the physician's ability to recognize the presence or absence of anemia, fever, and jaundice. Academic Emergency Medicine, 7(2), pp.146-156.

Institute of Public Health Nutrition (IPHN) (2007). National Strategy for Colour Prevention and Control in Bangladesh. Ministry of Health and Family Welfare Government of the People's Republic of Bangladesh.

Kaplan, R.M., Burke, J.M., Terrill, T.H., Miller, J.E., Getz, W.R., Mobini, S., Valencia, E., Williams, M.J., Williamson, L.H., Larsen, M. and Vatta, A.F. (2004). Validation of the FAMACHA@ eye color chart for detecting clinical anemia in sheep and goats on farms in the southern United States. Veterinary parasitology, 123(1), pp.105-120.

Mahieu, M., Arquet, R., Kandassamy, T., Mandonnet, N. and Hoste, H. (2007). Evaluation of targeted drenching using Famacha $\subseteq$ method in Creole goat: reduction of anthelmintic use, and effects on kid production and pasture contamination. Veterinary parasitology, 146(1), pp.135-147.

Nardone, D.A., Roth, K.M., Mazur, D.J. and McAfee, J.H. (1990). Usefulness of physical examination in detecting the presence or absence of anemia. Archives of internal medicine, 150(1), pp.201-204.

Sheth, T.N., Choudhry, N.K., Bowes, M. and Detsky, A.S. (1997). The relation of conjunctival pallor to the presence of anemia. Journal of general internal medicine, 12(2), pp.102-106.

Shiffman, D., 2008. Learning Processing: A Beginner's Guide to Programming Images. Animation, and Interaction, Burlington, USA.

Van Wyk, J.A. and Bath, G.F. (2002). The FAMACHA system for managing haemonchosis in sheep and goats by clinically identifying individual animals for treatment. Veterinary research, 33(5), pp.509-529.

Walter, T., Klein, J.C., Massin, P. and Erginay, A., 2002. A contribution of image processing to the diagnosis of diabetic retinopathy-detection of exudates in color fundus images of the human retina. IEEE transactions on medical imaging, 21(10), pp.1236-1243.

World Health Organization (WHO) (1991). Prevention and Management of Severe Colour in Pregnancy (Report of a Technical Working Group), (WHO/FHE/MSM/93.5). 